Case Report

\title{
Case report: Uncommon Sulfamethoxazole crystalluria
}

\author{
Vincent Castiglione*, Etienne Cavalier, Romy Gadisseur \\ Clinical Chemistry Department, CHU de Liège, University of Liège, Belgium
}

A R T I C L E I N F O

\section{Keywords:}

Sulfamethoxazole

Crystalluria

Drug: adverse effect

Acute renal failure

Urine microscopy

\section{Case report}

We report seven Caucasian patients with unusual Sulfamethoxazole crystalluria on random urine sediment analysis. Among them, there were 3 female and 4 male, aged between 54 and 86 years-old, admitted to hospital for different bacterial infections: three had urinary tract infection, two had osteitis and two developed sepsis. All patients had several comorbidities, but no pathology in common.

The unidentified crystals were discovered thanks to an automated urine analyzer SediMAX ${ }^{\circ}$ (Menarini, Florence, Italy). However, the analyzer wasn't able to identify them correctly because of their various uncommon shapes (Fig. 1). In each sample, most of the crystals were birefringent on polarized microscopy. Urine $\mathrm{pH}$ varied from 5.0 to 6.5. A Fourier Transform Infrared Spectrophotometry analysis was performed on the dried urine residue. In all cases, the main metabolite of Sulfamethoxazole, N-Acetylsulfamethoxazole (NASM) was identified.

The administrated doses of Sulfamethoxazole varied from $800 \mathrm{mg}$ to $4800 \mathrm{mg}$ per day, during 5 to $>30$ days. The crystalluria were observed between 1 and 28 days after the treatment's beginning. Lower cotrimoxazole doses were associated to crystals with rectangular shapes, while higher doses were associated to bigger and more irregular crystals. An eGFR (MDRD) decrease was associated to crystalluria onset in three patients. Three other patients had no creatinine increase, while it wasn't assessed in the last one. The urine strip analysis revealed no anomaly in common between patients.

After diagnosis, cotrimoxazole was replaced by another antibiotherapy. No crystalluria were observed after stopping treatment, excepted in one patient. Two of the three patients who developed acute renal failure resolved within 15 days after the end of Sulfamethoxazole antibiotherapy, while one didn't.
Supplementary data about infrared analysis protocol and detailed patients parameters are available in Data in Brief [1].

\section{Discussion}

Only 4 papers have reported a total of 5 cases of NASM crystalluria associated to acute renal failure yet [2]. It is the first time that a larger number of patients with new and very heterogeneous shapes of $\mathrm{N}$ Acetyl-Sulfamethoxazole crystalluria is reported and confirmed by infrared spectroscopy.

Drug-induced kidney failure is well-known, but is also probably under-evaluated. Even if it was not the main cause of renal failure, we suspect Sulfamethoxazole could have played a role in these cases.

These NASM crystalluria are more susceptible to appear with high doses of cotrimoxazole, hypovolemia and pre-existing renal failure [3]. Other risk factors included acid urinary $\mathrm{pH}$, low daily urine volume and high urine concentration in both Sulfamethoxazole and NASM [4]. Hypoalbuminemia is another risk factor present in five of our patients $[2,3]$.

Therefore, the treatment of NASM precipitation should include hydratation, dosage adaptation in regard of renal function, or even stopping treatment [5]. Urine alkalinization $(\mathrm{pH}>7.0)$ is also possible. The evaluation of the real role and prevalence of NASM in acute renal failure require further studies. The monitoring of renal function and the screening of urine sediment of patients at risk should enhance the detection of crystalline nephropathy. Infrared analysis is mandatory to confirm crystal's composition.

\footnotetext{
Abbreviation:NASM, N-Acetyl-Sulfamethoxazole

* Corresponding author at: CHU Sart Tilman, University of Liège, Clinical Chemistry department, Avenue de l'Hôpital, n1, 4000 Liège, Belgium.

E-mail address: V.Castiglione@doct.uliege.be (V. Castiglione).
} 


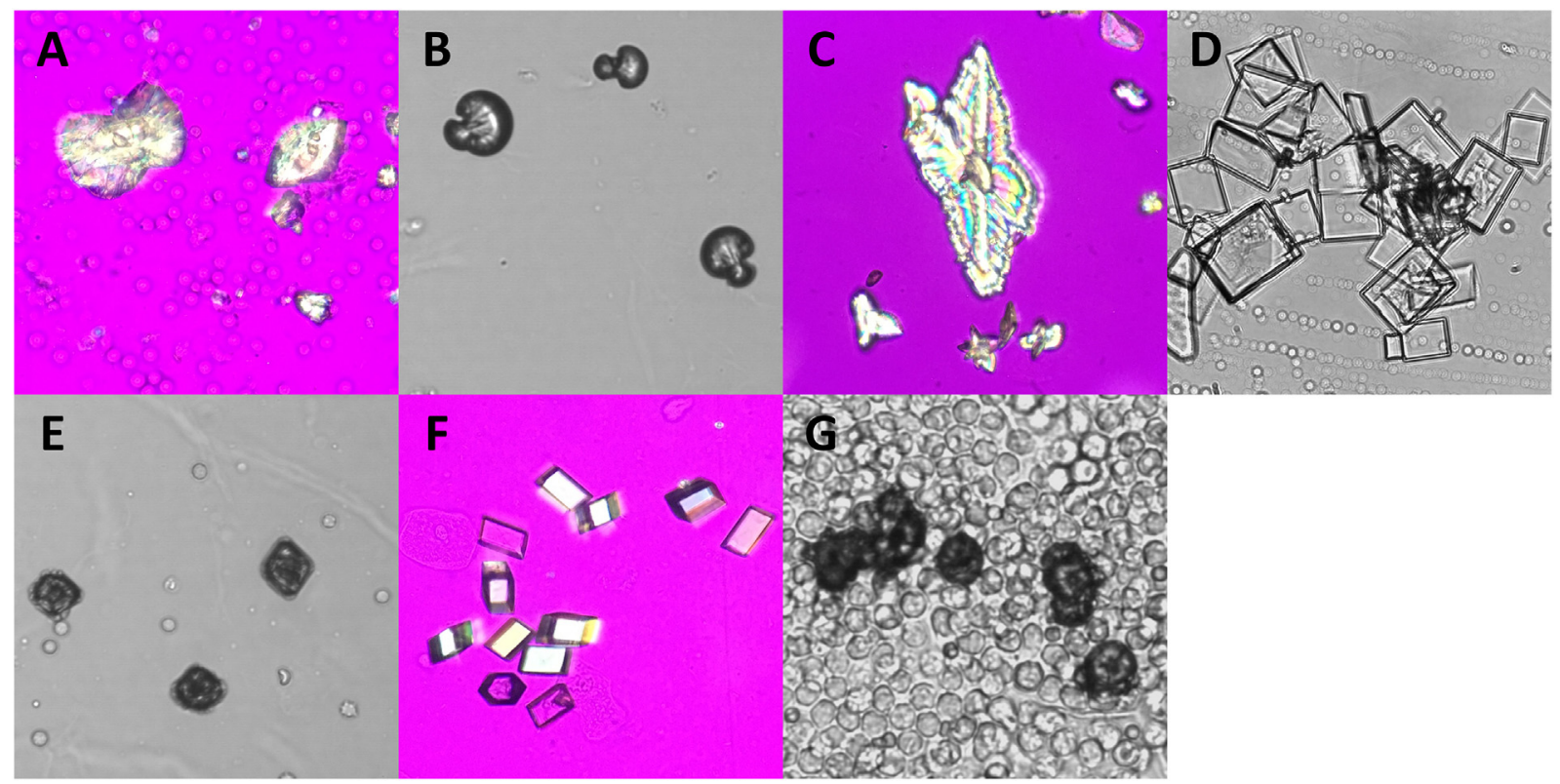

Fig. 1. N-Acetyl-Sulfamethoxazole crystalluria.

Various N-Acetyl-Sulfamethoxazole crystal shapes, including asymmetric lozenge (A), mushrooms (B), flower-like (C), thin rectangles (D), truncated lozenges (E), thick parallelepipeds (F) and spheroids (E). Pictures on bright-field (B, D, E, G) and polarized light (A, C, F) microscopy. Original magnification: 400 ×. All these crystals were identified in different patients. Please refer to the Data in Brief article for more information about crystalluria parameters.

\section{Informed consent}

This study is in accordance with the Helsinki Declaration and with our Ethical Committee.

\section{Acknowledgement, disclosure and fundings}

The authors declare to have no disclosure.

This research did not receive any specific grant from funding agencies in the public, commercial, or not-for-profit sectors.

\section{References}

[1] V. Castiglione, E. Cavalier, R. Gadisseur, Clinical Data on Rare Sulfamethoxazole
Crystalluria Assessed by Fourier Transform Infrared Spectrophotometry, Data Br, (2018) (In press).

[2] N. Buchanan, Sulphamethoxazole, hypoalbuminaemia, crystalluria, and renal failure, Br. Med. J. 2 (1978) 172 http://www.ncbi.nlm.nih.gov/pubmed/678832, Accessed date: 22 November 2016.

[3] M.A. Perazella, Crystal-induced acute renal failure, Am. J. Med. 106 (1999) 459-465, http://dx.doi.org/10.1016/S0002-9343(99)00041-8.

[4] O.S. Alfthan, K. Liewendahl, Investigation of sulfonamide crystalluria in man, Scand. J. Urol. Nephrol. 6 (1972) 44-46 http://www.ncbi.nlm.nih.gov/pubmed/5057618.

[5] F. de Liso, G. Garigali, C. Ferraris Fusarini, M. Daudon, G.B. Fogazzi, How to identify sulfamethoxazole crystals in the urine, Clin. Chim. Acta 452 (2016) 106-108, http:// dx.doi.org/10.1016/j.cca.2015.11.006. 